DR. RUPESH RAINA (Orcid ID : 0000-0003-3892-8376)

Article type : Original

The role of continuous renal replacement therapy in the management of acute kidney injury associated with sinusoidal obstruction syndrome following hematopoietic cell transplantation

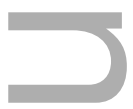

Rupesh Raina ${ }^{2^{\star}}$, Ghada A Abusin ${ }^{1 *}$, Prashant Vijayaraghavan ${ }^{7}$, Jeffery J. Auletta ${ }^{3}$, Linda Cabral ${ }^{4}$, Hasan Hashem ${ }^{3}$, Beth A. Vogt ${ }^{5}$, Kenneth R. Cooke ${ }^{6}$ and Rolla F AbuArja $^{3}$

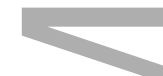

*Rupesh Raina and Ghada A Abusin are first authors

${ }^{1}$ Pediatric Bone Marrow Transplant, University of Michigan, Ann Arbor, Michigan, USA

${ }^{2}$ Pediatric Nephrology, Akron Children's Medical Center; Northeast Ohio Medical University, Akron, Ohio, USA

${ }^{3}$ Pediatric Blood and Marrow Transplant Program, Nationwide Children's Hospital, Ohio State University College of Medicine; Columbus, Ohio, USA

${ }^{4}$ Pediatric Blood and Marrow Transplant Program, Rainbow Babies and Children's Hospital, Cleveland, Ohio, USA

${ }^{5}$ Pediatric Nephrology, Rainbow Babies and Children's Hospital, Cleveland, Ohio, USA ${ }^{6}$ Department of Oncology, Pediatric Blood and Marrow Transplantation Program, The Sidney Kimmel Cancer Center, Johns Hopkins University, Baltimore, Maryland, USA

This is the author manuscript accepted for publication and has undergone full peer review but has not been through the copyediting, typesetting, pagination and proofreading process, which may lead to differences between this version and the Version of Record. Please cite this article as doi: $\underline{10.1111 / \text { petr.13139 }}$

This article is protected by copyright. All rights reserved 
The work was performed at Rainbow Babies and Children's Hospital, Cleveland, Ohio, USA

${ }^{7}$ Cleveland Clinic Akron General/Akron Nephrology Associates, Akron, Ohio, USA

Corresponding author

Rupesh Raina MD

Consultant Nephrologist

Adult-Pediatric Kidney Disease/Hypertension

Department of Nephrology

Cleveland Clinic Akron General and

Akron Children's Hospital, Akron, Ohio, USA

Phone: $330-543-8950$

Fax: $330-543-3980$

rraina@akronchildrens.org

raina@akronnephrology.com

Running title: Role of CRRT in AKI and SOS following HCT

\title{
Authorship Statement
}

All the authors are involved in all stages of the study and manuscript preparation.

\begin{abstract}
Background: Maintaining fluid balance pre- and post- myeloablative hematopoietic cell transplant (MA-HCT) is essential and usually requires frequent administration of diuretics. Hepatic sinusoidal obstructive syndrome is potentially life threatening, especially when associated with AKI and multi-organ failure. This study describes six patients who developed AKI-associated SOS and diuretic-resistant fluid overload who subsequently underwent continuous renal replacement therapy using standardized management guidelines for fluid balance post-HCT.
\end{abstract}


Materials and methods: Retrospective chart review of HCT patients between September 2011 and October 2013 at a tertiary care children's hospital.

Results: Thirty-four patients underwent MA-HCT in the study period. Six patients had SOS complicated by diuretic-resistant FO and underwent CRRT. Defibrotide was used in three patients. Median time on CRRT was 10.5 days. Sixty six percent $(\mathrm{N}=4 / 6)$ of patients had full resolution of SOS symptoms with mortality rate of $34 \%(\mathrm{~N}=2 / 6)$. Among patients who had full recovery of SOS symptoms, one patient developed AKI, end stage renal diseases and underwent kidney transplantation 34-months post-HCT.

Conclusions: Thus, out of 6 included patients, 2 died and 1 developed ESRD with only $50 \%(\mathrm{~N}=3 / 6)$ good outcome. Use of a standardized, evidence-based fluid-balance protocol and early initiation of CRRT for HCT related AKI/SOS was associated with good outcomes.

\section{Key words:}

Hematopoietic cell transplant; fluid overload; acute kidney injury; continuous renal replacement therapy; sinusoidal obstruction syndrome Introduction

Acute kidney injury (AKI) is a common comorbidity in pediatric patients following hematopoietic cell transplantation (HCT), with an incidence ranging from $11-84 \%$ of these, $5-10 \%$ of patients may require renal replacement therapy (RRT). [1-3] Overall survival of children after HCT decreases significantly with increasing severity in AKI within the first 100 days post-HCT. [2, 3] Sinusoidal obstruction syndrome (SOS) is a potentially life-threatening, early post-HCT complication. [4] Although mild SOS resolves without long-term sequelae in most patients, SOS associated with multi-organ failure (MOF) has very high mortality rates, exceeding $80 \%$ despite aggressive supportive therapy.

Management of fluid balance is critical in the HCT patient. In particular, sodium and fluid restriction and diuretic use are cornerstones to the prevention of fluid overload in SOS-associated AKI. [5] Continuous renal replacement therapy (CRRT) has emerged as a treatment modality in critically-ill children with AKI and fluid overload (FO). [6] Standardized guidelines for initiating CRRT in the setting of SOS are not established. 
Severe SOS requires a multidisciplinary approach for optimal patient outcomes. $[5,7]$ The Pediatric HCT, Nephrology and Critical Care teams developed institutional guidelines for managing AKI and FO in HCT patients. This report describes the index case that initiated the development of fluid management protocol attempting to standardize a clinical approach for five more patients who underwent CRRT as a supportive care modality in managing their severe SOS.

\section{Materials and methods}

Data for this report were obtained from a retrospective chart review of HCT patients between September 2011 and October 2013 at Rainbow Babies \& Children's Hospital. All patients who developed SOS, diuretic-resistant fluid overload and AKI were included for further description. The University Hospitals Case Medical Center Institutional Review Board approved this study.

Definitions

Day of hematopoietic cell infusion was defined as day zero (D0). Baseline weight $(\mathrm{kg})$ was defined as either the hospital admission weight (wt.) if the patient was admitted directly for transplant or the patient's weight on the first day of preparative conditioning regimen if the patient was admitted prior to HCT for any other medical reasons.

Baseline height was the height used to calculate the body surface area (BSA) for dosing calculations related to HCT. Baseline serum creatinine (SCr) was defined as the lowest $\mathrm{SCr}$ in the three-month span preceding the start of conditioning regimen.

SOS was defined according to modified Seattle criteria; which are the presence of either hyperbilirubinemia (total bilirubin $\geq 2 \mathrm{mg} / \mathrm{dL}$ ) before post-transplant day 21 , or tender hepatomegaly, weight gain ( $>5 \%$ from baseline) or ascites. [4,8]. When present, liver ultra-sound demonstrating reversal of portal venous blood flow was used in support of the diagnosis of SOS. AKI was defined and stratified using Kidney Disease: Improving Global Outcomes (KDIGO) Table $1(2,18)$.

Patients' fluid status was assessed by twice daily weight measurements, strict fluid input and output recordings, and daily renal function panel. Fluid overload was defined as the percentage of fluid accumulation by dividing the cumulative balance in 
liters by patient's baseline weight and multiplying by $100 \%$. We then used the cutoff value of $>5 \%$ of fluid accumulation as a definition of fluid overload (16):

$\% \mathrm{FO}$ (weight) $=($ Weight - Admit weight $\left.) \times(\text { Admit weight })^{-1}\right] \times 100$
$\%$ FO (input/output $)=\left[(\right.$ Total fluid input in liters-Total fluid output in liters $) \times\left(\right.$ Admit weight ${ }^{-}$
$\left.{ }^{1}\right] \times 100$

Criteria for CRRT initiation included all the following: SOS, AKI-F, and diureticresistant FO $>10 \%$ (16). CRRT was initiated within 8- 12 hours of SOS diagnosis. The diuretic resistance is defined as fluid retention (24 hours after initiation of Furosemide) despite the use of appropriate doses of frusemide in patient with $\mathrm{FO}>5 \%$, with decreased renal function and clinical evidence of reduced and delayed peak concentrations of loop diuretics in the tubular fluid as evident by suboptimal urine output of less than $0.5 \mathrm{ml} / \mathrm{kg} / \mathrm{hr}$. after $4-6 \mathrm{hrs}$. of last frusemide infusion $(16,17)$.

\section{CRRT protocol}

CRRT blood flow rates ranged from $4-5 \mathrm{ml} / \mathrm{kg} / \mathrm{min}$. Initial fluid removal rates were determined by the PICU and Nephrology physicians and adjusted as tolerated, ranging from 1-2 m/ $/ \mathrm{kg} / \mathrm{hr}$. Standard citrate anticoagulation was used to maintain post-filter ionized calcium (iCa) between 0.25 and $0.35 \mathrm{mmol} / \mathrm{L}$ and pre-filter iCa between 1.1 and $1.3 \mathrm{mmol} / \mathrm{L}$. Replacement fluid calculated as follows $(2000 \mathrm{ml} / \mathrm{h} \times \mathrm{BSA}) \times\left(1.73 \mathrm{~m}^{2}\right)^{-1}$. In patients receiving defibrotide, efforts were made to keep INR $<1.5$ and platelets $>30,000 / \mathrm{mm}^{3}$.

\section{Statistical Analysis}

For all patients who underwent allogeneic or autologous HCT procedure as described above, medical records were used to abstract demographics, diagnoses, survival, clinical and laboratory data pertaining to markers of inflammation, as well as pulmonary, renal, cardiac, and hepatic function. Statistical analysis of specific comparisons between the two patient groups of survivors and non-survivors was performed using the two-sided Fisher's exact test. $P$-values less than 0.05 were regarded as significant. 


\section{Results}

Thirty-four patients (age 1-26 years) received allogeneic $(n=30)$ and autologous $(n=4)$ HCT between September 2011 and October 2013. Thirty-one patients received single $\mathrm{HCT}$; one patient with beta-thalassemia major underwent three matched unrelated donor (MUD) HCTs due to repeated graft failure; one patient with severe aplastic anemia underwent two MUD HCTs for primary graft failure; and one patient received two autologous tandem transplants for atypical Teratoid Rhabdoid tumor of the brain.

Fourteen patients had normal kidney function around engraftment period. Twenty patients $(59 \%)$ developed AKI, further stratified according to AKI per KDIGO-AKIN guideline Stage I (8/20), Stage II (6/20) and stage III (6/20) occurring 14-60 days after the first HCT. Six patients fulfilled criteria for SOS (all had tender hepatomegaly, rapid weight gain and ascites) that was complicated by AKI (grade III AKI per KDIGO) and diuretic-resistant FO and subsequently received CRRT. All six patients received busulfan-based Myeloablative (MA) conditioning regimen; and busulfan doses were based on test-dose pharmacokinetics for all patients. Table 2 summarizes clinical demographics of this case series. The abdominal compartment pressures (measured $<12$ hrs.) at the initiation of CRRT are mentioned in Table 2.

\section{Patient 1 (Index-case)}

Two-year-old female who underwent MA-HCT for rare immune deficiency (Bare lymphocyte syndrome type 2) was diagnosed with SOS associated with hypotension and coagulopathy 19 days following allogeneic HCT. Due to active hemorrhage, defibrotide was not administered. Her course was complicated by stage III AKI per KDIGO; and diuretic-resistant FO. She was started on CRRT when she reached $40 \%$ fluid overload (72 hours after reaching 10\% fluid overload). Unfortunately, her clinical condition rapidly deteriorated to MOF, and she died 18 days after SOS diagnosis (D37 post-HCT).

Following this index case, an algorithm was developed to manage fluid overload in HCT patients (Figure 1). Five subsequent patients with SOS and stage III AKI: and 
developed diuretic-resistant FO and were started on CRRT within 24 hours of meeting the criteria. Defibrotide was used in 3 patients. Reasons for not receiving defibrotide in the other patients were: unavailability of the drug (Patient 2) and retinal hemorrhage (Patient 6) (Table 2).

Median CRRT duration was 10.5 days and total CRRT days for all patients were 55 days. Citrate anticoagulation was well tolerated, and only one patient developed citrate gap, which resolved after adjusting citrate infusion rate. No side effects related to metabolic alkalosis, acidosis, or hypocalcemia were observed. No serious hemorrhagic events occurred. All five patients had complete resolution of SOS signs and symptoms as well as marked improvement in renal function, with 5 days' mean recovery time. One patient died on D97 post-HCT due to systemic Cytomegalovirus and Aspergillosis despite full recovery of renal function and SOS. Three of 5 patients had complete recovery of kidney function post-CRRT; one patient developed AKI-E and underwent kidney transplantation 34-months post-HCT (Patient-6) (Table 2). The overall length of stay (in days) in those without and with AKI for the whole cohort was $63.1 \pm 47.3$ vs. 86 $\pm 45.2(P<0.05)$. The overall crude mortality of the patient who received allogeneic or autologous HCT without or with AKI was $0 \%$ vs. $20 \%(P<0.05)$.

\section{Discussion}

Incidence of AKI after HSCT ranges between $25-50 \%$, and the incidence of renal replacement therapy (RRT) is $5-18 \%$. In our cohort, $59 \%$ of patients developed AKI (1460 days after $\mathrm{HCT}$ ). Fluid overload (>20\%) at the time of CRRT initiation, grade III AKI per KDIGO; and multisystem organ failure ( $>3$ systems), were the risk factors associated with the mortality in these patients.

With increased applications of HCT for different disease indications, evaluation and treatment of HCT-related complications has become increasingly important to mitigate early transplant-related mortality and improve outcomes. [5, 8] SOS can be a devastating complication after HCT $[4,8]$. Several studies have demonstrated that up to $50 \%$ of patients with SOS develop renal or pulmonary dysfunction [9]. Outcomes for these patients are poor as mortality rates reach as high as $84 \%$ by D100 post-HCT [10]. For patients who meet criteria for SOS (Baltimore or modified Seattle), supportive care efforts are centered on aggressive fluid management with special attention to 
maintaining intravascular volume; so kidney perfusion is maintained. [5] In this regard, it is critical to avoid exacerbating AKI with aggressive diuresis or rapid removal of ascetic fluid. HCT recipients often require intravenous parenteral nutrition, frequent administration of blood products, and multiple nephrotoxic medications, all of which may worsen FO, further contributing to AKI [1, 2, 4, 8, 9]. Therefore, management of AKI and FO in HCT patients is particularly challenging; and when FO $>20 \%$ or is refractory to conventional pharmaceutical diuresis, CRRT may need to be considered. This study showed that early initiation of CRRT and aggressive diuresis to prevent fluid overload seems to be necessary, but not sufficient for pediatric SCT patients with AKI. Also, early CRRT may blunt the inflammatory response and prevent need for intubation or increase likelihood of extubation. CRRT requires dialysis catheter placement, sedation and ICU care to monitor, prevent and manage potential hemorrhagic events that are inherent to SOS pathophysiology (refractory thrombocytopenia and coagulopathy due to hepatic dysfunction) and potentially associated with the use of defibrotide [10-12,16].

Our study was based on the initial publication by Goldstein et which showed that early initiation of diuretic in $\mathrm{HCT}(\mathrm{FO}>5 \%)$ and or CRRT (FO>10\%) prevents worsening of FO and may improve the survival (42\%) of HCT patients with AKI. Using and implementing the algorithm in our cohort of HCT patients with SOS showed marked improvement in mortality from $90 \%$ to $50 \%$. $(13,16)$

In our cohort of the patients who did not receive defibrotide, 1/3 was alive without ESRD. Of the patients who received defibrotide, 2/3 was alive without ESRD. One could argue that the use of defibrotide and not the CRRT protocol have been the cause of the better outcomes. However, defibrotide is the only available FDA approved therapy for SOS that is accompanied by either renal or pulmonary dysfunction after HCT. Yet not all patients can receive defibrotide in timely manner for medical reasons, including active bleeding, concomitant use of systemic anticoagulation or fibrinolytic therapy or known hypersensitivity to the drug. $[10,11]$ It is proposed that defibrotide is not the sole reason for improved outcome, but early CRRT/FO is a paramount for SOS treatment. Traditionally defibrotide has improved D100 post-HCT survival in patients with SOS and MOF with up to $40 \%$ of patients treated were on mechanical ventilation, dialysis or both at the time of drug initiation [10]. 
While defibrotide has emerged as a therapeutic option for many patients with SOS, managing fluid and acid-base balance in the context of AKI remains a significant challenge in pathogenesis of SOS. In our cohort the marked improvement in mortality from $90 \%$ to $34 \%$ is due to early recognition of patients at risk for development of AKI post-HCT transplant by way of standardized methods of staging and prevention of the occurrence of $\mathrm{AKL}$ is paramount to improve the outcomes of an otherwise life-saving therapy. Early initiation of CRRT may be a useful modality to prevent progressive fluid overload and maintain electrolyte and acid base balance in patients with $\mathrm{FO}$ and $\mathrm{AKI}$ following SOS.

Skeen's et al found significant variability in the diagnosis and management of SOS between HCT and Critical Care providers. [7]. The current work, presents our institutional experience with collaborative efforts between Pediatric HCT, Nephrology and Critical Care providers using a clinical evidence-based algorithm for fluid management and early CRRT initiation in pediatric HCT patients with SOS, AKI and FO (Figure 1). Following the unfavorable outcome of Patient 1 it instituted this standardized clinical approach to pediatric HCT patients with SOS-associated AKI and FO. Based on this approach, five subsequent patients were started on CRRT within 24 hours of meeting algorithm-based criteria. Following adoption of the algorithm, the observation of overall improvement in renal and liver functions in this cohort was realized. It is believed this outcome occurred because of adherence to the algorithm for fluid management and early CRRT initiation. In addition, it shows that regional citrate anticoagulation seemed to be safe form of anticoagulation in HCT patients with SOS, despite the potential risk of citrate toxicity associated with liver impairment. [13] None of the patients developed hemorrhagic events, metabolic acidosis, alkalosis, hypocalcemia or other electrolyte abnormalities. From this one can conclude that citrate anticoagulation is feasible and was not associated with apparent side effects in the population studied.

This study finds the incidence of $\mathrm{AKI}$ in the cohort to be high (59\%), which may reflect the ability to identify patients with $\mathrm{AKI}$ using the KDIGO criteria, or prior fluid management approach.

Regarding the diagnosis of AKI, KDIGO agreed with pRIFLE /RIFLE $92 \%$ of the time. All three definitions correlate well with outcomes, however KDIGO was preferable 
for AKI stage discrimination. pRIFLE used the Swartz formula but in this setting, a kinetic formula's that predicts true endogenous kidney function is needed, fluctuations in creatinine production, and fluid balance rather than steady-state GFR estimator (18). Measuring endogenous eGFR is expensive and complex, considerably outweighing their reliability and making them unsuitable for routine use in the patients with AKI following HCT. Authors feels AKIN has a ddiagnostic timeframe and more practical by using serum creatinine and urine output as a surrogate on daily basis rather that static eGFR which is dependent on heights or baseline creatinine values which are likely to be missing. Regardless of any definitions severe AKI is associated with higher mortality and longer length of stay in the ICU.

A new concept of renal angina index (RAI) to improve the prediction of acute kidney injury in critically ill children by Goldstein et al together with KDIGO classification and renal biomarkers may provide a clinically feasible and applicable methodology to identify HCT patient at risk of severe AKI lasting beyond functional injury. $(16,17) \mathrm{A}$ unified AKI definition in subset of HCT cohort is needed and it is recommended by the authors that the nephrology and oncology community continue to work toward this goal.

Flores et al. used the Prospective Pediatric CRRT Registry to analyze 51 patients who underwent HCT and required CRRT. The authors noted that the \%FO at CRRT initiation was relatively low $(<12 \%)$ and similar between survivors and nonsurvivors, suggesting that increased mortality risk was related to factors other than excessive fluid accumulation. [14] By contrast, Sutherland et al. examined \%FO at initiation of CRRT in 297 critically-ill children and demonstrated that fluid overload is independently associated with mortality. [15] These contradictory reports illustrate the paucity of data available regarding the optimal timing of CRRT. Wallhult et al. recently published the review for management of SOS utilizing a multidisciplinary approach to care. [5] This emphasized the role of nursing in early detection of the symptoms associated with SOS and timely treatment, with the goal of improving the outcome. [5] Intensive nursing with alleviating clinical barriers/efforts to get twice daily weights in the ICU setting, daily \%FO calculation, strict intake and output charting including insensible loses should be incorporated into clinical care of patients with HCT. The authors concur with the guidelines and add to it our recommendation for early initiation of CRRT. 
This analysis is limited by all the weaknesses inherent with a retrospective data collection and small number of patients. Clearly, prospective trial would appear indicated to confirm these findings.

\section{Conclusion}

Use of a standardized, evidence-based approach involving careful fluid management for patients undergoing HCT is essential. Early recognition of patients at risk for development of $A K I$ post-transplant and prevention of the occurrence of $A K I$ is paramount to improve the outcomes of an otherwise life-saving therapy. Renal support with early initiation of CRRT as an adjunct to enhance-renal function, modifying fluid balance (despite FO>20\%), acid-base balance and control solute level may help in reducing mortality and morbidity of patients with FO and AKI following SOS. CRRT with citrate regional anticoagulation is safe and well tolerated in patients with SOS. Findings emphasize the need for multi-disciplinary approach to early recognition and management of $\mathrm{AKI}$ in patients with HCT-related SOS to improve outcomes (decreasing overall mortality from $90 \%$ to $50 \%$ ). Nephrologists and Oncologists should work together to identify at risk patients and to prevent development of AKI by initiating therapy early to improve outcomes. Future large prospective studies are needed to confirm our findings.

\section{Funding}

This research received no specific grant from any funding agency in the public, commercial, or not-for-profit sectors.

\section{References}

1. Didsbury MS, Mackie FE, Kennedy SE. A systematic review of acute kidney injury in pediatric allogeneic hematopoietic stem cell recipients. Pediatric transplantation. 2015;19(5):460-70. Epub 2015/05/13. doi: 10.1111/petr.12483. PubMed PMID: 25963934.

2. Kizilbash SJ, Kashtan CE, Chavers BM, Cao Q, Smith AR. Acute Kidney Injury and the Risk of Mortality in Children Undergoing Hematopoietic Stem Cell Transplantation. Biology of blood and marrow transplantation : journal of the American Society for Blood and Marrow 
Transplantation. 2016;22(7):1264-70. Epub 2016/04/02. doi: 10.1016/j.bbmt.2016.03.014. PubMed PMID: 27034153; PubMed Central PMCID: PMCPMC5178146.

3. Rajpal JS, Patel N, Vogel RI, Kashtan CE, Smith AR. Improved survival over the last decade in pediatric patients requiring dialysis after hematopoietic cell transplantation. Biology of blood and marrow transplantation : journal of the American Society for Blood and Marrow Transplantation. 2013;19(4):661-5. Epub 2012/12/26. doi: 10.1016/j.bbmt.2012.12.012. PubMed PMID: 23266833.

4. Coppell JA, Richardson PG, Soiffer R, Martin PL, Kernan NA, Chen A, et al. Hepatic venoocclusive disease following stem cell transplantation: incidence, clinical course, and outcome. Biol Blood Marrow Transplant. 2010;16(2):157-68. Epub 2009/09/22. doi: 10.1016/j.bbmt.2009.08.024. PubMed PMID: 19766729; PubMed Central PMCID: PMCPMC3018714.

5. Wallhult E, Kenyon M, Liptrott S, Mank A, Ni Chonghaile M, Babic A, et al. Management of veno-occlusive disease: the multidisciplinary approach to care. European journal of haematology. 2017;98(4):322-9. Epub 2016/12/20. doi: 10.1111/ejh.12840. PubMed PMID: 27992075.

6. Goldstein SL, Currier H, Graf C, Cosio CC, Brewer ED, Sachdeva R. Outcome in children receiving continuous venovenous hemofiltration. Pediatrics. 2001;107(6):1309-12. Epub 2001/06/05. PubMed PMID: 11389248.

7. Skeens MA, McArthur J, Cheifetz IM, Duncan C, Randolph AG, Stanek J, et al. High Variability in the Reported Management of Hepatic Veno-Occlusive Disease in Children after Hematopoietic Stem Cell Transplantation. Biology of blood and marrow transplantation : journal of the American Society for Blood and Marrow Transplantation. 2016;22(10):1823-8. Epub 2016/08/09. doi: 10.1016/j.bbmt.2016.07.011. PubMed PMID: 27496218.

8. Naples JC, Skeens MA, Auletta J, Rangarajan H, Abu-Arja R, Horwitz E, et al. Anicteric veno-occlusive disease after hematopoietic stem cell transplantation in children. Bone marrow transplantation. 2016;51(1):135-7. Epub 2015/09/15. doi: 10.1038/bmt.2015.208. PubMed PMID: 26367232.

This article is protected by copyright. All rights reserved 
9. Carreras E, Diaz-Beya M, Rosinol L, Martinez C, Fernandez-Aviles F, Rovira M. The incidence of veno-occlusive disease following allogeneic hematopoietic stem cell transplantation has diminished and the outcome improved over the last decade. Biology of blood and marrow transplantation : journal of the American Society for Blood and Marrow Transplantation. 2011;17(11):1713-20. Epub 2011/06/29. doi: 10.1016/j.bbmt.2011.06.006. PubMed PMID: 21708110.

10. Richardson PG, Cooke KR, Krishnan AY. CAHO Dec Supp Cover Strategies for the Management of Hepatic Veno-Occlusive Disease in Patients Undergoing Bone Marrow Transplant. Strategies. 2015.

11. Richardson PG, Corbacioglu S, Ho VT, Kernan NA, Lehmann L, Maguire C, et al. Drug safety evaluation of defibrotide. Expert Opin Drug Saf. 2013;12(1):123-36. Epub 2012/12/12. doi: 10.1517/14740338.2012.749855. PubMed PMID: 23228043.

12. Dalle JH, Giralt SA. Hepatic Veno-Occlusive Disease after Hematopoietic Stem Cell Transplantation: Risk Factors and Stratification, Prophylaxis, and Treatment. Biology of blood and marrow transplantation : journal of the American Society for Blood and Marrow Transplantation. 2016;22(3):400-9. Epub 2015/10/04. doi: 10.1016/j.bbmt.2015.09.024. PubMed PMID: 26431626.

13. Wonnacott R, Josephs B, Jamieson J. CRRT Regional Anticoagulation Using Citrate in the Liver Failure and Liver Transplant Population. Crit Care Nurs Q. 2016;39(3):241-51. Epub 2016/06/03. doi: 10.1097/cnq.0000000000000118. PubMed PMID: 27254640.

14. Flores FX, Brophy PD, Symons JM, Fortenberry JD, Chua AN, Alexander SR, et al. Continuous renal replacement therapy (CRRT) after stem cell transplantation. A report from the prospective pediatric CRRT Registry Group. Pediatric nephrology (Berlin, Germany). 2008;23(4):625-30. Epub 2008/01/30. doi: 10.1007/s00467-007-0672-2. PubMed PMID: 18228045.

15. Sutherland SM, Goldstein SL, Alexander SR. The prospective pediatric continuous renal replacement therapy (ppCRRT) registry: a critical appraisal. Pediatr Nephrol. 2014;29(11):206976. Epub 2013/08/29. doi: 10.1007/s00467-013-2594-5. PubMed PMID: 23982708.

This article is protected by copyright. All rights reserved 
16. Michael M, Kuehnle I, Goldstein SL. Fluid overload and acute renal failure in pediatric stem cell transplant patients. Pediatric nephrology (Berlin, Germany). 2004;19(1):91-5. Epub 2003/11/25. doi: 10.1007/s00467-003-1313-z. PubMed PMID: 14634863.

17. Basu RK, Zappitelli M, Brunner L, Wang Y, Wong HR, Chawla LS, et al. Derivation and validation of the renal angina index to improve the prediction of acute kidney injury in critically ill children. Kidney Int. 2014;85(3):659-67. Epub 2013/09/21. doi: 10.1038/ki.2013.349. PubMed PMID: 24048379; PubMed Central PMCID: PMCPMC4659420.

18. Sutherland SM, Byrnes JJ, Kothari M, Longhurst CA, Dutta S, Garcia P, et al. AKI in hospitalized children: comparing the pRIFLE, AKIN, and KDIGO definitions. Clin J Am Soc Nephrol. 2015;10(4):554-61. Epub 2015/02/05. doi: 10.2215/cjn.01900214. PubMed PMID: 25649155; PubMed Central PMCID: PMCPMC4386245.

This article is protected by copyright. All rights reserved 
Table 1. The KDIGO classification/staging system of acute kidney injury ${ }^{a}$

Stage $\mathrm{SCr}$

UO

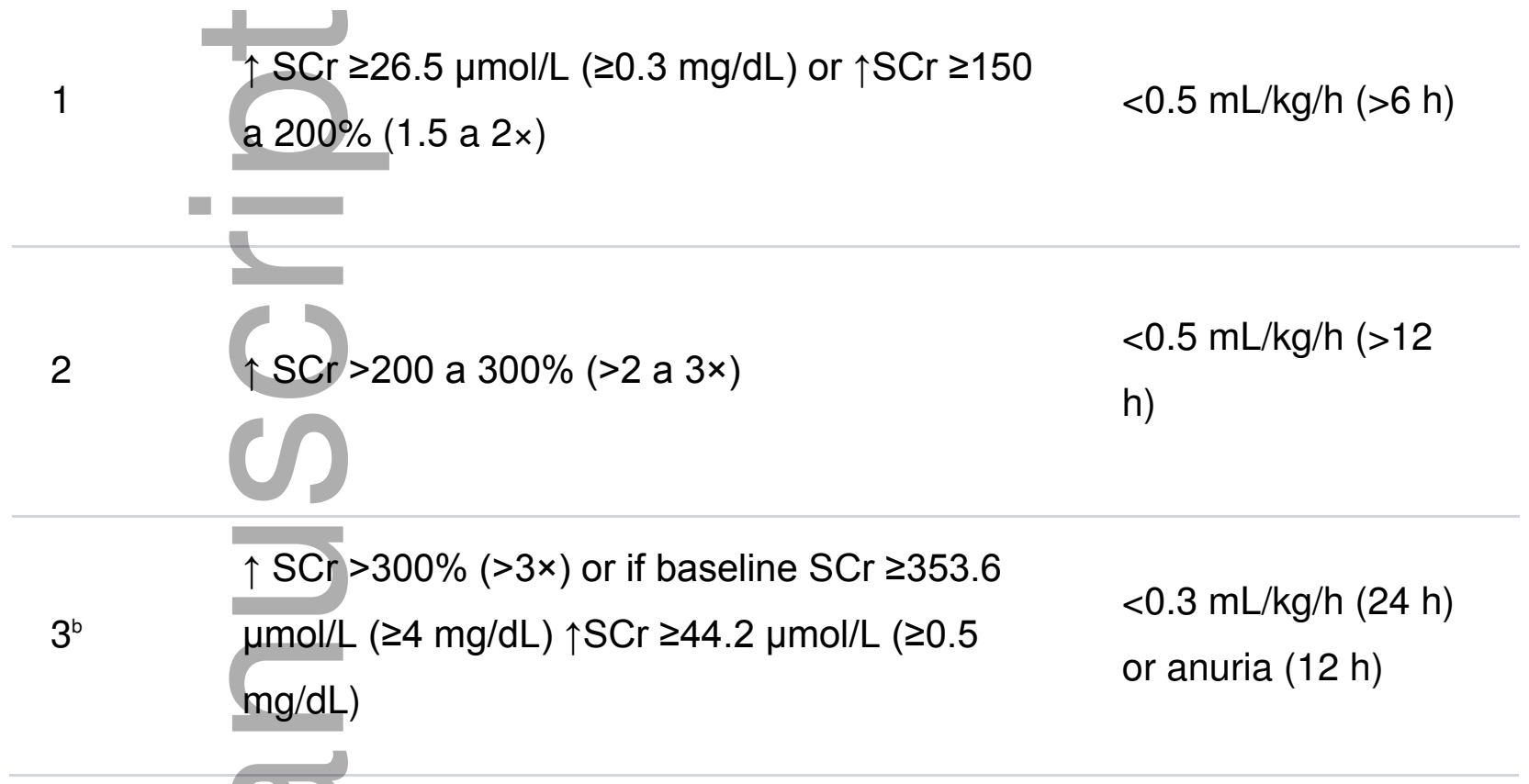

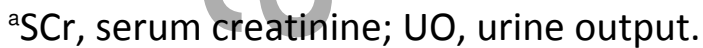

${ }^{b}$ Stage 3 also includes patients requiring RRT independent of the stage (defined by SCr and/or UO) they are in at the moment they initiate RRT.

This article is protected by copyright. All rights reserved 
Table 2 Patient Demographics

\begin{tabular}{|c|c|c|c|c|c|c|}
\hline Variables & Patient 1 & Patient 2 & Patient 3 & Patient 4 & Patient 5 & Patient 6 \\
\hline $\begin{array}{l}\text { Age } \\
\text { (years)/gender/ }\end{array}$ & 2/F/BLS-2 & 6/M/MDS & 16/M/CML & 12/F/AML & 17/M/MDS & 15/M/AML \\
\hline diagnosis & & & & & & \\
\hline $\begin{array}{l}\text { Conditioning } \\
\text { regimen }\end{array}$ & Bu/Cy/ATG & Bu/Cy & Bu/Cy/ATG & Bu/Cy/ATG & Bu/Cy/ATG & Bu/Cy/ATG \\
\hline Stem cell source & $\mathrm{BM}$ & PBSC & CB & BM & PBSC & BM \\
\hline $\begin{array}{l}\text { SOS onset post } \\
\text { HCT (days) }\end{array}$ & & 11 & 18 & 11 & 19 & 20 \\
\hline $\begin{array}{l}\text { CRRT initiation } \\
\text { after SOS } \\
\text { diagnosis }\end{array}$ & 72-96 hrs. & $9 \mathrm{hrs}$. & $10 \mathrm{hrs}$. & $8.5 \mathrm{hrs}$. & $11 \mathrm{hrs}$. & 9.5 hrs. \\
\hline $\begin{array}{l}\text { \%FO at the onset } \\
\text { of CRRT }\end{array}$ & 40 & 20 & 5 & 20 & 12 & 8 \\
\hline $\begin{array}{l}\text { ACP at onset of } \\
\text { CRRT }\end{array}$ & 32 & 14 & 12 & 12 & 18 & 10 \\
\hline $\begin{array}{l}\text { Defibrotide } \\
\text { Time to start }\end{array}$ & No & No & Yes & Yes & Yes & No \\
\hline $\begin{array}{l}\text { Defibrotide after } \\
\text { SOS diagnosis }\end{array}$ & & - & $24 \mathrm{hrs}$. & 26 hrs. & 20 hrs. & - \\
\hline Total & & & & & & \\
\hline $\begin{array}{l}\text { bilirubin/creatini } \\
\text { ne at initiation of }\end{array}$ & $14 / 3.1$ & $1.8 / 0.63$ & $6.3 / 1.52$ & $1.6 / 1.23$ & $8.3 / 1.45$ & $3.2 / 2.15$ \\
\hline $\operatorname{CRRT}(\mathrm{mg} / \mathrm{dl})$ & & & & & & \\
\hline $\begin{array}{l}\text { CRRT duration } \\
\text { (days) }\end{array}$ & 0.5 & 6 & 9 & 15 & 12 & 12 \\
\hline $\begin{array}{l}\text { CRRT } \\
\text { Anticoagulation }\end{array}$ & Heparin & Citrate & Citrate & Citrate & $\begin{array}{l}\text { Citrate/ } \\
\text { Heparin }\end{array}$ & $\begin{array}{l}\text { Citrate } \\
\text { /Heparin }\end{array}$ \\
\hline Outcome & $\begin{array}{l}\text { Died } \\
(\text { Day +37) }\end{array}$ & Alive & Alive & Alive & $\begin{array}{l}\text { Died } \\
(\text { Day }+97)\end{array}$ & $\begin{array}{l}\text { Alive/ } \\
\text { ESRD }\end{array}$ \\
\hline
\end{tabular}

AML: acute myeloid leukemia, ATG: anti-thymoglobulin, BLS-2: Bare lymphocyte syndrome type 2, BM: bone marrow, Bu: busulfan, ACP in mm (Normal $<20 \mathrm{mmHG}$ ) Abdominal Compartment Pressure, CRRT: continuous renal replacement therapy, CB: cord blood, CY: cyclophosphamide, F: female, MDS: myelodysplastic syndrome, M: male, PB: peripheral blood stem cells, SOS: sinusoidal obstruction syndrome

This article is protected by copyright. All rights reserved 
Figure 1. Algorithm to the management of fluid status in the patients undergoing

hematopoietic cell transplant. The clinical algorithm that was developed after an index case (Patient

1 in Table 2).

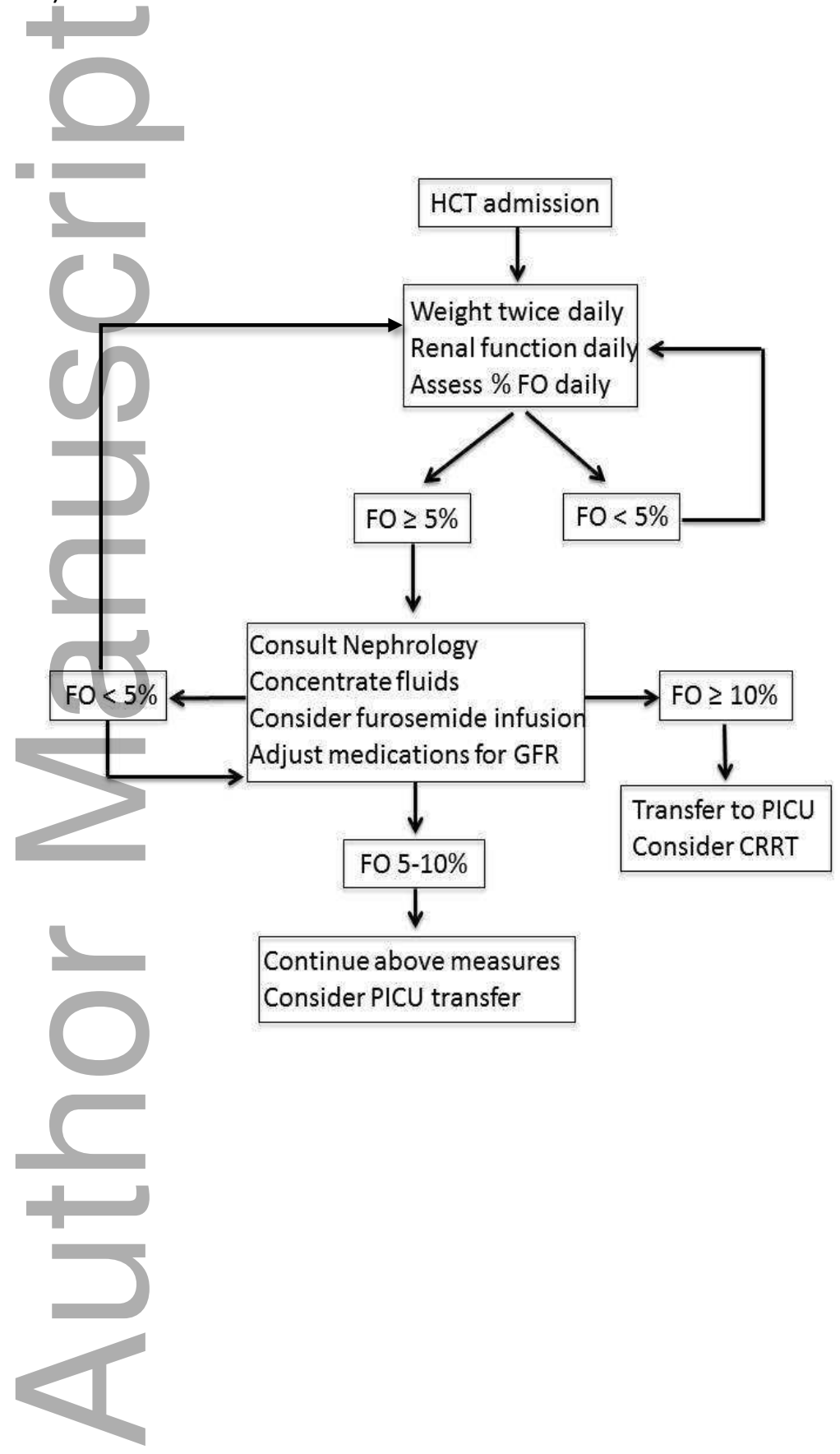

This article is protected by copyright. All rights reserved 